\title{
Foreword
}

\section{Practice Management: \\ Successfully Guiding Your Group into the Future}

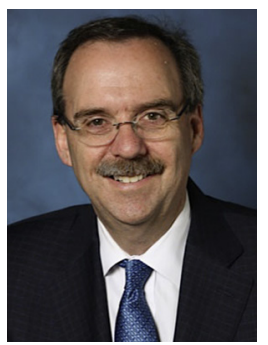

Lee A. Fleisher, MD, FACC, FAHA Consulting Editor

Medicine is both a noble profession and social good, but is also a business. The future of health care is clearly focused on the value proposition. Within that context, the field of anesthesiology is actively engaged in defining its value. In this issue of the Anesthesiology Clinics, the editors have focused on three issues of practice management: operating room management, quality and reporting, and the value proposition. As we attempt to articulate our value to the payers and "employers," such as hospitals, ambulatory surgery centers, and health systems, the information in these articles should prove very useful.

This issue was proposed and edited by two thought-leaders in this arena. Dr Abouleish is a professor of Anesthesiology in the division of pediatric anesthesia at the University of Texas Medical Branch in Galveston, Texas. While at UTMB, he completed his MBA at University of Houston Clear Lake. Dr Abouleish's research interest is the economics of anesthesia care, and specifically, the measurement and benchmarking of clinical productivity in anesthesiologists and anesthesiology groups. He is on several American Society of Anesthesiologists (ASA) committees, including the Committee on Economics and the Committee on Practice Management, for which he is a past chair. Dr Stead is CEO and Founder of the Stead Health Group. He received his MBA from UCLA. He is currently Vice President for Professional Affairs for the ASA. He has extensive experience in health care coding and 
payment expertise. Together, they have assembled an amazing group of leaders in our specialty to describe different models of transformation of clinical practice.

Lee A. Fleisher, MD, FACC, FAHA Perelman School of Medicine University of Pennsylvania 3400 Spruce Street, Dulles 680

Philadelphia, PA 19104, USA

E-mail address:

Lee.Fleisher@uphs.upenn.edu 